\title{
Dynamic Valuation of Weather Derivatives under Default Risk
}

\section{Wolfgang Karl Härdle* Maria Osipenko*}

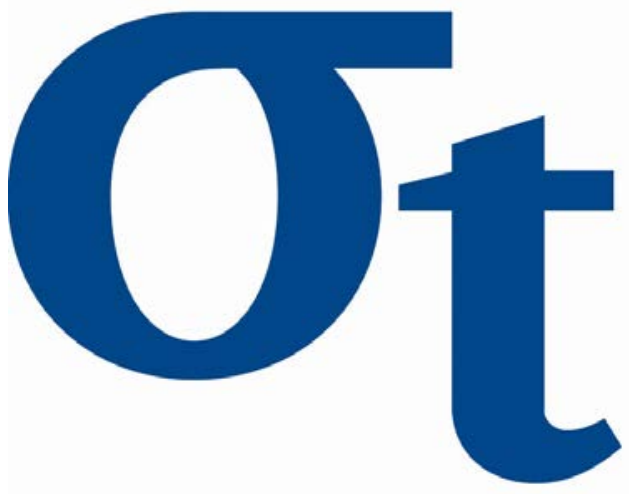

*Humboldt-Universität zu Berlin, Germany 


\title{
Dynamic Valuation of Weather Derivatives under Default Risk*
}

\author{
Wolfgang Karl Härdle ${ }^{1}$ and Maria Osipenko ${ }^{\dagger 2}$ \\ ${ }^{1}$ CASE - Centre for Applied Statistics and Economics, \\ Humboldt-Universität zu Berlin, Germany \\ ${ }^{2}$ School of Business and Economics, Humboldt-Universität zu Berlin
}

\begin{abstract}
Weather derivatives are contingent claims with payoff based on a pre-specified weather index. Firms exposed to weather risk can transfer it to financial markets via weather derivatives. We develop a utility-based model for pricing baskets of weather derivatives in over-the-counter markets under counterparty default risk. In our model, agents maximise the expected utility of their terminal wealth, while they dynamically rebalance their weather portfolios over a finite investment horizon. Via partial market clearing, we obtain semi-closed forms for the equilibrium prices of weather derivatives and for the optimal strategies of the agents. We give an example on how to price rainfall derivatives on selected stations in China in the universe of a financial investor and a weather exposed crop insurer.
\end{abstract}

Keywords: derivative securities, asset pricing models MSC 2010 Classification: 91G20, 91B25

\footnotetext{
*Authors acknowledge financial support of Deutsche Forschungsgemeinschaft through the CRC 649: Economic Risk.

${ }^{\dagger}$ Correspondence Address: School of Business and Economics, Humboldt-Universität zu Berlin, Unter den Linden 6, 10099, Germany. Email: mariaosipenko@yahoo.de.
} 


\section{Introduction}

Weather derivatives (WDs) are contingent claims with payoffs determined by future weather events as temperature, snowfall, and rainfall. Hedging with WDs reduces exposure to the weather conditions and stabilises the profits of a weather exposed agent. Firms operating in energy, tourism, agriculture, and insurance sectors use WDs to hedge their weather risks. Peréz-González and Yun (2013) show that weather risk management with WDs leads to an increase in firm value. Moreover, WDs are also attractive for a purely financial investor as their payoffs are acyclic, uncorrelated with financial assets, and, therefore, contribute to portfolio diversification.

Frequently, the structure of agent's weather exposure is complicated, such that, it is more beneficial to purchase a basket of WDs on several underlying weather indices rather than on a single one to manage weather risks in an optimal way. For a financial investor, positions in multiple WDs strengthen the positive portfolio diversification effect. To increase the hedging efficiency and to achieve a higher degree of portfolio diversification, both weather exposed business and financial investors are, therefore, interested in holding a portfolio of multiple WDs. While valuing baskets of such derivatives, market participants should also account for the dependence in the underlying weather indices.

We develop a simple utility-based model for pricing such baskets of customised WDs on multiple dependent underlyings. With our model, we primary address pricing WDs in overthe-counter (OTC) markets, where fewer interested agents transfer their specific non-financial weather risks among themselves through creating and trading customized weather dependent claims. We allow agents to rebalance their portfolios (or renegotiate the contracts) at some predetermined time points in their finite investment horizon. Dynamic portfolio optimisation 
under partial market clearing or zero-net-supply condition for all WDs determines their equilibrium prices.

We choose equilibrium pricing approach based on utility maximisation for several reasons. First, we anticipate that the OTC transactions of WDs involve a limited number of participants. Thus, the interaction of their individual supply and demand functions will give the equilibrium price. Further, due to the market incompleteness standard arguments imposing existence of a unique pricing measure or a perfect replication strategy fail, see Heath et al. (1992). Models based on pure weather dynamics impose restrictive assumptions in order to choose the appropriate equivalent martingale measure, see Alaton et al. (2002), Benth et al. (2007), and López Cabrera et al. (2013). In our model, involved agents construct WD securities in zero net supply, thus, they complete the WD market. Moreover, the chosen utility-based pricing approach naturally reflects the risk attitudes of each agent and facilitates the derivation of explicit prices for WDs in a multi-period framework.

Most previous works on utility-based WD pricing either fail to price derivatives on multiple dependent underlyings simultaneously (Carmona and Diko (2005), Lee and Oren (2010), and Leobacher and Ngare (2011)) or they impose restrictive assumptions on the dynamics of the underlying weather indices (Horst and Müller (2007), Chaumont et al. (2006)). Consumption based model of Cao and Wei (2004) prices multiple WDs in an extended pure exchange economy of Lucas (1978) on the macroeconomic level and is not feasible for a limited number of weather market participants and a short contract duration typical for OTC markets. We contribute to this literature by providing a utility-based model for pricing baskets of customised WDs on multiple dependent underlyings. In our framework, market participants account for possible dependence between the weather indexes that determine the payoffs of their weather portfolios. Applicability of our pricing model is not restricted to 
any particular type of WDs. Various kinds of OTC WDs can be priced in our framework.

Our further contribution is the introduction of counterparty default risk into the pricing model for WDs. The importance of counterparty default risk for pricing financial derivatives was addressed, for example, in Hull and White (1995), Jarrow and Turnbull (1995), and Wu and Chung (2010). We show that the introduction of a non-zero issuer default probability significantly depresses the demand for WDs through correcting expectations of future portfolio payoffs in utility terms downwards.

We illustrate our approach on pricing rainfall derivatives. These derivatives can be used to hedge agricultural volumetric risks, see Musshoff et al. (2010). Traditionally, volumetric risk hedging in agriculture is taken over by the crop insurance as Glauber et al. (2002). Therefore, crop insurer frequently face indirect losses caused by rainfall surplus/deficit through its impact on crop production. Whenever the weather exposed income of such an insurer depends on the rainfall outcomes in a number of geographical sites where the insured farmers are operating, a combination of the rainfall derivatives on these sites should be used for hedging the risks.

We give the pricing example for China. Chinese farmers are exposed to pronounced weather risks as Turvey and Kong (2010). According to The World Bank (2007) the existing agricultural insurance schemes are too expensive for Chinese agricultural producers. Trading WDs can play an important role in transferring a part of the weather exposure from the crop insurer to financial markets and so make such crop insurance contracts affordable for Chinese farmers.

Our empirical analysis addresses the effects of increasing investment horizon, default risk, market volatility and capital costs on the demand and on the supply for WDs and illustrates WD price movements. 
The structure of the paper is as follows. In section II we, first, derive the multi-asset dynamic pricing model for WDs in absence of any default risk. Next, we extend the model to account for the counterparty default risk and a possibility of an alternative financial investment. Section III shows an example on pricing rainfall derivatives using historical rainfall data of China and features a discussion of further applications. In section 4 we summarise our results.

\section{Dynamic Pricing Model for Weather Derivatives}

In this section, we construct a dynamic pricing model for WDs with multiple assets. First, we introduce our market design and notation. Then, we derive the equilibrium prices for WDs without any default risk. Next, we study the implications of the counterparty default risk and of an alternative financial investment.

\subsection{Assumptions and Notation}

1. Assets. There are WDs on $S$ weather indices (at different geographical sites and/or on different weather events) that are priced at times $t=0,1, \ldots, T-1$. At $T$ the payoff of each WD is determined and the cash settlement takes place.

Besides the WDs, a risk free asset $B_{t}$ with a known constant per period return $r_{t}$ is available. Trading with $B_{t}$ is not restricted in any way, that is, unlimited borrowing and lending at the interest $r_{t}$ in each $t$ is allowed.

2. Agents. There are $J+1$ heterogeneous market participants, indexed by $i$, with risk preferences described by the exponential utility function of the form $U_{i}(x)=-\exp \left(-a_{i} x\right)$, where $a_{i}>0$ is the risk aversion of agent $i$. All agents have the same multi-period 
investment horizon of length $T$. They invest at $t=0$ and they consume their terminal wealth at $t=T$. At $t=1, \ldots, T-1$ agents rebalance their weather portfolios and renegotiate the prices for WDs. All agents are endowed with initial wealth of zero monetary units. We distinguish between $J$ buyers, indicated by subscript $j, j=1, \ldots, J$, who hedge weather exposure of their random bounded income $I_{j}$ which they get at time $T$, and a purely financial investor, indicated by subscript $m$, who issues WDs. Each buyer holds a basket of WDs on the relevant weather indices to hedge weather caused fluctuations in her profits. The issuer holds positions in all $S$ WDs. At time $t$, the portfolio of agent $i$ includes $\alpha_{i, t}=\left(\alpha_{i, t, 1}, \ldots, \alpha_{i, t, S}\right)^{\top}$ shares of the corresponding WDs and $\beta_{i, t}$ shares of the asset $B_{t}$. Both $\alpha_{.,}$and $\beta_{.,}$are real valued, that is, all assets are perfectly divisible and short sales are allowed. We denote the value of $i$ th agent's portfolio at time $t$ as $V_{i, t}$, where $V_{i, t}=\alpha_{i, t}^{\top} W_{t}+\beta_{i, t} B_{t}$. In each period $t$ of the investment horizon, agents maximise their expected utility of the terminal wealth with the available WDs and attain their demand and supply for the WDs. That is, in each period $t<T$ every agent $i$ determines her self-financing trading strategy $\left(\alpha_{i, t+1}, \beta_{i, t+1}\right)_{t=0,1, \ldots, T}^{\top}$, in particular, she constructs the optimal hedging portfolio given the state of the system at time t. Partial market clearing with respect to WDs determines the equilibrium prices for the WDs.

We assume, that the information structure is perfect and symmetric. The information set at time $t, \mathcal{F}_{t}$, represents the growing information level of the agents up to time $t$. The expectation with respect to $\mathcal{F}_{t}$ is denoted as $\mathrm{E}_{t}(\cdot)$.

3. State. The observable state of the system at time $t$, denoted by $\mathcal{W}_{t}$, contains all realisations of relevant system variables at time $t$. The evolution to the next state $\mathcal{W}_{t+1}$ is characterised by the conditional distribution function $\Phi_{t}(w \prime, w)=\operatorname{Pr}\left(\mathcal{W}_{t+1} \leq\right.$ 
$\left.w \prime \mid \mathcal{W}_{t}=w\right)$. We assume that each transition function $\Phi_{t}$ for $t=0, \ldots, T-1$ satisfies the Feller property Stokey et al. (1989).

Each agent $i \in J$ is faced with the following discrete time stochastic control system:

$$
V_{i, t+1}=g_{i, t}\left\{V_{i, t},\left(\alpha_{i, t, s}\right)_{s \in S}, \mathcal{W}_{i, t}\right\}, \quad t=0,1, \ldots, T
$$

where $V_{i, t}$ incorporates portfolio value of agent $i$ with $V_{i, 0}=0 .\left(\alpha_{i, t, s}\right)_{s \in S}$ are controls of the agent in the system (1). The continuous law of motion $g_{i, t}: \mathbb{R}^{S+1} \times \mathbb{R}^{\left|S_{j}\right|} \times \mathbb{R}^{\left|S_{j}\right|} \mapsto$ $\mathbb{R}^{\left|S_{j}\right|+1}$ maps to the next wealth state of the agent.

\subsection{Pricing WDs without Default Risk}

We now derive a dynamic pricing model for WDs with multiple assets under the market design introduced by assumptions 1 to 3 . For the moment, we assume that there is no default risk.

The terminal wealth of buyer $j$ at $T$ is her profit $\Pi_{j, T}$ :

$$
\begin{aligned}
\Pi_{j, T} & =I_{j}+\alpha_{j, T}^{\top} W_{T}+\beta_{j, T} B_{T} \\
& =I_{j}+V_{j, T}
\end{aligned}
$$

with $I_{j}$ being the real-valued income at time $T$ that depends on some of the weather indices

entering the final payoff $W_{T} \cdot \beta_{j, T} B_{T}$ and $\alpha_{j, T}^{\top} W_{T}$ are the payoffs of the risk free asset and of the basket of the WDs; together they constitute $V_{j, T}$, the terminal portfolio value of buyer $j$. Let $\alpha_{j, t+1: T}$ denote trading strategies of agent $j$ from $t+1$ to $T$. Portfolio choice problem 
of buyer $j$ in each $t=0,1, \ldots, T-1$ is:

$$
\begin{gathered}
\max _{\alpha_{j, t+1: T} \in \mathbb{R}^{S \times(T-t)}} \mathrm{E}_{t}\left\{U_{j}\left(\Pi_{j, T}\right)\right\} \\
\text { s.t. } \alpha_{j, t+1}^{\top} W_{t}+\beta_{j, t+1} B_{t}-V_{j, t}=0 .
\end{gathered}
$$

with $V_{j, 0}=0$ and $U_{j}\left(\Pi_{j, T}\right)=-\exp \left(-\alpha_{j} \Pi_{j, T}\right)$. That is, in each period of the investment horizon buyer $j$ maximises the expected utility of her terminal wealth with respect to all future trading strategies, subject to a self-financing portfolio.

The terminal wealth of investor $m$ at $T$ is:

$$
\Pi_{m, T}=-\alpha_{m, T}^{\top} W_{T}+\beta_{m, T} B_{T}=V_{m, T}
$$

with $\alpha_{m, T}^{\top} W_{T}$ and $\beta_{m, T} B_{T}$ being the payoffs of the WDs portfolio and the risk free asset respectively. Investor's portfolio choice problem in each $t=0,1, \ldots, T-1$ is:

$$
\begin{gathered}
\max _{\alpha_{m, t+1: T} \in \mathbb{R}^{S \times(T-t)}} \mathrm{E}_{t}\left\{U_{m}\left(\Pi_{m, T}\right)\right\} \\
\text { s.t. } \alpha_{m, t+1}^{\top} W_{t}-\beta_{m, t+1} B_{t}+V_{m, t}=0 .
\end{gathered}
$$

with $V_{m, 0}=0$ and $U_{m}\left(\Pi_{m, T}\right)=-\exp \left(-\alpha_{m} \Pi_{m, T}\right)$. In $t<T$ investor $m$ maximises the expected utility of her terminal wealth with respect to all future trading strategies, subject to a self-financing portfolio.

Note, that under assumptions 1 to 3 , the constraints in the optimization problems 3 and 5 are nonempty, compact and continuous in $V_{.,}$and $\mathcal{W}$., $U .(\cdot)$ is continuous and bounded, and transition function $\Phi$ is Feller (by assumption). Thus, the continuous law of motion $g_{\cdot, \cdot}$ ensures the existense of optimal solutions to the problems above and the continuity of the 
value function (see Stokey et al. (1989),p.62).

Following Pennacchi (2008), we solve the multi-period portfolio choice problems (3) and (5) using dynamic programming. Let $J_{i, t}\left(V_{i, t}, \alpha_{i, t+1: T}, \mathcal{W}_{i, t}\right)$ denote the expected utility of agent $i$ in time $t$. It depends on the agent's current portfolio value $V_{i, t}$, the current state $\mathcal{W}_{i, t}$, and the trading strategies $\alpha_{i, t+1: T}$ in the time span $t+1$ to $T$ (here indicated by subscript $t+1: T)$. Let $J_{i, t}^{*}\left(V_{i, t}, \mathcal{W}_{i, t}\right)$ be the expected utility maximised with respect to the strategies of agent $i$ from $t+1$ on. Formally, in each $t J_{i, t}^{*}\left(V_{i, t}, \mathcal{W}_{i, t}\right)$ is obtained by:

$$
J_{i, t}^{*}\left(V_{i, t}, \mathcal{W}_{i, t}\right)=\max _{\alpha_{i, t+1: T} \in \mathbb{R}^{S \times(T-t)}}\left\{J_{i, t}\left(V_{i, t}, \alpha_{i, t+1: T}, \mathcal{W}_{i, t}\right)\right\}
$$

According to the principle of dynamic programming the expected utility of agent $i$ in $t$ can be rewritten as:

$$
J_{i, t}\left(V_{i, t}, \alpha_{i, t+1}, \mathcal{W}_{i, t}\right)=\mathrm{E}_{t}\left[J_{i, t+1}^{*}\left\{V_{i, t+1}\left(\alpha_{i, t+1}\right), \mathcal{W}_{i, t+1}\right\}\right]
$$

Combining (6) and (7), we obtain the recursive expression for $J_{i, t}^{*}\left(V_{i, t}, \mathcal{W}_{i, t}\right)$ :

$$
J_{i, t}^{*}\left(V_{i, t}, \mathcal{W}_{i, t}\right)=\max _{\alpha_{i, t+1} \in \mathbb{R}^{S}} \mathrm{E}_{t}\left[J_{i, t+1}^{*}\left\{V_{i, t+1}\left(\alpha_{i, t+1}\right), \mathcal{W}_{i, t+1}\right\}\right]
$$

with the boundary condition at $T$

$$
J_{i, T}^{*}\left(V_{i, T}, \mathcal{W}_{i, T}\right)=U_{i}\left(I_{i}+\alpha_{i, T}^{\top} W_{T}+\beta_{i, T} B_{T}\right)
$$

and with $\beta_{i, T} B_{T}=V_{i, T}-\alpha_{i, T}^{\top} W_{T}$.

Starting with $t=T-1$ and using (8), we recursively find the supply and the demand at 
$t$ for all the WDs, denoted in vector form as:

$$
W_{t}^{i}\left(\alpha_{i, t+1}\right)=\left\{W_{t, 1}^{i}\left(\alpha_{i, t+1}\right), \ldots, W_{t, S}^{i}\left(\alpha_{i, t+1}\right)\right\}^{\top}, \quad i=j, m
$$

Imposing a partial market clearing for WD securities (zero net supply), $\sum_{j=1}^{J} \alpha_{j, t+1, s}^{*}=$ $\alpha_{m, t+1, s}^{*}$, delivers the equilibrium prices $W_{t}=W_{t}^{m}\left(\alpha_{m, t+1}^{*}\right)$ for all $t=0, \ldots, T-1$, where * identifies the optimal values. The specific forms of buyer's demand and investor's supply are given in Propositions 1 and 2 below.

PROPOSITION 1. Let the utility function of the buyer $j$ be of the exponential form with risk aversion $a_{j}>0$. Then, under assumptions 1 to 3 her reverse demand for WD $s$, $W_{t, s}^{j}\left(\alpha_{j, t+1}\right)$, and her optimal utility level $J_{j, t}^{*}\left(V_{j, t}, \mathcal{W}_{j, t}\right)$ are given recursively by:

$$
\begin{aligned}
W_{t, s}^{j}\left(\alpha_{j, t+1}\right) & =\frac{\mathrm{E}_{t}\left\{\exp \left(-a_{j} \alpha_{j, t+1}^{\top} W_{t+1} R^{T-(t+1)}\right) \Theta_{j, t+1} W_{t+1, s}\right\}}{\mathrm{E}_{t}\left\{\exp \left(-a_{j} \alpha_{j, t+1}^{\top} W_{t+1} R^{T-(t+1)}\right) \Theta_{j, t+1}\right\} R}, \\
J_{j, t}^{*}\left(V_{j, t}, \mathcal{W}_{j, t}\right) & =-\exp \left(-a_{j} V_{j, t} R^{T-t}\right) \Theta_{j, t}, \\
\Theta_{j, t} & =\exp \left(a_{j} R^{T-t} \alpha_{j, t+1}^{* \top} W_{t}\right) \mathrm{E}_{t}\left\{\exp \left(-a_{j} R^{T-(t+1)} \alpha_{j, t+1}^{* \top} W_{t+1, s}\right) \Theta_{j, t+1}\right\},
\end{aligned}
$$

for $t=0, \ldots, T-1, \Theta_{j, T}=\exp \left(-a_{j} I_{j}\right)$ and $R=1+r$.

Proof. See the Appendix.

PROPOSITION 2. Let the utility function of the investor be of the exponential form with risk aversion $a_{m}>0$. Then, under assumptions 1 to 3 her reverse supply for WD $s$, 
$W_{t, s}^{m}\left(\alpha_{m, t+1}\right)$, and her optimal utility level $J_{m, t}^{*}\left(V_{m, t}, \mathcal{W}_{m, t}\right)$ are given recursively by:

$$
\begin{aligned}
W_{t, s}^{m}\left(\alpha_{m, t+1}\right) & =\frac{\mathrm{E}_{t}\left[\exp \left\{a_{m}\left(\alpha_{m, t+1}^{\top} W_{t+1, s} R^{T-(t+1)}\right) \Theta_{m, t} W_{t+1, s}\right\}\right]}{\mathrm{E}_{t}\left\{\exp \left(a_{m} \alpha_{m, t+1}^{\top} W_{t+1} R^{T-(t+1)}\right) \Theta_{m, t}\right\} R}, \\
J_{m, t}^{*}\left(V_{m, t}, \mathcal{W}_{m, t}\right) & =-\exp \left(-a_{m} V_{m, t} R^{T-t}\right) \Theta_{m, t}, \\
\Theta_{m, t} & =\exp \left(-a_{m} \alpha_{m, t+1}^{* \top} W_{t} R^{T-t}\right) \mathrm{E}_{t}\left\{\exp \left(a_{m} R^{T-t+1} \alpha_{m, t+1}^{* \top} W_{t+1}\right) \Theta_{m, t+1}\right\}
\end{aligned}
$$

for $t=0, \ldots, T-1, \Theta_{m, T}=1$ and $R=1+r$.

Proof. The proof is very similar to the proof of Proposition 1 and is omitted.

The results above show that the reverse demand and supply for the sth WD at time $t$ are determined by the interaction of the terms proportional to the marginal expected utility of the next period as well as future expected utilities in $\Theta_{i, t+1}$ that embrace optimal future trading behaviour, $\alpha_{i, t+1: T}$. Moreover, the reverse demand and supply are both influenced by the capital costs or risk-free rate $r_{t}$. Changes in $r_{t}$ will, therefore, influence the resulting WD prices.

The expressions obtained are of a semi-closed form, since the expectations entering (11) and (14) have, in general, no explicit representation and have to be computed numerically using, for example, Monte Carlo techniques.

\subsection{Default risk}

As we already mentioned, OTC derivatives are subject to the counterparty default risk or credit risk as termed in Golden et al. (2007). In this section we incorporate the issuer default risk into the pricing model for WDs. 
As in Golden et al. (2007), we consider dichotomous default risk modelled by a payoff proportion parameter $\lambda_{t}$ with values in $\{0,1\}$. While $\lambda_{t}=1$ means that the counterparty fully meets its obligations in $t, \lambda_{t}=0$ inherits the situation where the counterparty does not perform at all. We assume that $\lambda_{t}$ is independent of all other random variables in the model, and $P\left(\lambda_{t+1}=0\right)=p_{t}, P\left(\lambda_{t+1}=1\right)=1-p_{t}$, where $p_{t}$ is the probability of investor's default at $t+1$. Parameters $\lambda_{t}$ and $p_{t}$ are known to the buyers by $t$. Since once the investor defaults, she is not going to meet any obligations in subsequent periods, if $\lambda_{t}=0$ we set $\lambda_{t+1: T}=0$.

Clearly, under the non-zero investor's default risk, the payoff vector of the WDs is a multiple of $\lambda_{T}$, that is, $\lambda_{T} W_{T}$. If the investor has met all her obligations up to period $t<T$, then the contribution of the next period WD prices is $\lambda_{t+1} W_{t+1}$. Hence, the $j$ th buyer's demand for WD $s$ in (11) has to be modified to account for the default risk in the following way:

$$
W_{t, s}^{j}\left(\alpha_{j, t+1}\right)=\frac{\mathrm{E}_{t}\left\{\exp \left(-a_{j} \alpha_{j, t+1}^{\top} \lambda_{t+1} W_{t+1} R^{T-(t+1)}\right) \tilde{\Theta}_{j, t+1} \lambda_{t+1} W_{t+1, s}\right\}}{\mathrm{E}_{t}\left\{\exp \left(-a_{j} \alpha_{j, t+1}^{\top} \lambda_{t+1} W_{t+1} R^{T-(t+1)}\right) \tilde{\Theta}_{j, t+1}\right\} R}
$$

with

$$
\begin{aligned}
\tilde{\Theta}_{j, t}= & \exp \left(a_{j} R^{T-t} \alpha_{j, t+1}^{* \top} W_{t}\right) \\
& \cdot \mathrm{E}_{t}\left\{\left(1-p_{t}\right) \exp \left(-a_{j} R^{T-(t+1)} \alpha_{j, t+1}^{* \top} \lambda_{t+1} W_{t+1, s}\right) \tilde{\Theta}_{j, t+1}+p_{t} \Theta_{j, T}\right\}
\end{aligned}
$$

and $\Theta_{j, T}=\exp \left(-a_{j} I_{j}\right)$.

To derive (17) we consider all probable outcomes of $\lambda_{t+1}$ at each $t$. Thus, in $T-1$, if $\lambda_{T-1}=1$ we solve the maximisation problem (3) with the payoff of the WDs scaled by $\lambda_{T}$ and equal to $\lambda_{T} W_{T}$. As a result, we find $\alpha_{j, T}^{*}>0, W_{T-1}>0$, and $J_{j, T-1}^{*}\left(V_{j, T-1}, \mathcal{W}_{T}\right)$ which 
is equal to:

$$
J_{j, T-1}^{*}\left(V_{j, T-1}, \mathcal{W}_{T-1}\right)=-\exp \left\{-a_{j} V_{j, T-1} R\right\} \Theta_{j, T-1}^{\prime}
$$

with

$$
\Theta_{j, T-1}^{\prime}=\exp \left\{a_{j} \alpha_{j, T}^{* \top} W_{T-1} R\right\} \mathrm{E}_{T-1}\left[\exp \left\{-a_{j}\left(\alpha_{j, T}^{* \top} \lambda_{T} W_{T}\right)\right\} \Theta_{j, T}\right]
$$

If $\lambda_{T-1}=0 \alpha_{j, T}^{*}=0, W_{T-1}=0$, and

$$
J_{j, T-1}^{*}\left(V_{j, T-1}, \mathcal{W}_{T-1}\right)=-\exp \left\{-a_{j} V_{j, T-1} R\right\} \Theta_{j, T-1}^{\prime}
$$

where $\Theta_{j, T-1}^{\prime}=\mathrm{E}_{T-1}\left(\Theta_{j, T}\right)$.

We move one period backwards to $T-2$. If now $\lambda_{T-2}=1$ we maximise:

$$
\begin{gathered}
J_{j, T-2}\left(V_{j, T-2}, \alpha_{j, T-1}, \mathcal{W}_{j, T-2}\right)=\mathrm{E}_{T-2}\left\{J_{j, T-1}^{*}\left(V_{j, T-1}, \mathcal{W}_{j, T-1}\right)\right\} \\
=\mathrm{E}_{T-2}\left[-\exp \left\{-a_{j} V_{j, T-1} R\right\} \tilde{\Theta}_{j, T-1}\right], \\
\tilde{\Theta}_{j, T-1}=\exp \left\{a_{j} \alpha_{j, T}^{* \top} W_{T-1} R\right\} \mathrm{E}_{T-1}\left[\left(1-p_{T-1}\right) \exp \left\{-a_{j}\left(\alpha_{j, T}^{* \top} \lambda_{T} W_{T}\right)\right\} \Theta_{j, T}+p_{T-1} \Theta_{j, T}\right] .
\end{gathered}
$$

Note, that the expectation in first line of $(22)$ is taken under the joint distribution of $\lambda_{T-1}$ and the other random variables of the model, and in the second line - only with respect to the joint distribution of the later ones. $J_{j, T-2}^{*}\left(V_{j, T-2}, \mathcal{W}_{j, T-2}\right)$ is in this case:

$$
J_{j, T-2}^{*}\left(V_{j, T-2}, \mathcal{W}_{j, T-2}\right)=-\exp \left\{-a_{j} V_{j, T-2} R\right\} \Theta_{j, T-2}^{\prime}
$$

with

$$
\Theta_{j, T-2}^{\prime}=\exp \left(a_{j} \alpha_{j, T-1}^{* \top} W_{T-2} R\right) \mathrm{E}_{T-2}\left[\exp \left\{-a_{j}\left(\alpha_{j, T-1}^{* \top} \lambda_{T-1} W_{T-1}\right)\right\} \tilde{\Theta}_{j, T-1}\right]
$$


If $\lambda_{T-2}=0$ then $\alpha_{j, T-1}^{*}=0, W_{T-2}=0$, and

$$
J_{j, T-2}^{*}\left(V_{j, T-2}, \mathcal{W}_{j, T-2}\right)=-\exp \left\{-a_{j} V_{j, T-2} R\right\} \Theta_{j, T-2}^{\prime}
$$

where $\Theta_{j, T-2}^{\prime}=\mathrm{E}_{T-2}\left(\Theta_{j, T}\right)$. Then, the following expectation reads:

$$
\mathrm{E}_{T-3}\left\{J_{j, T-2}^{*}\left(V_{j, T-2}, \mathcal{W}_{j, T-2}\right)\right\}=\mathrm{E}_{T-3}\left[-\exp \left\{-a_{j} V_{j, T-2} R\right\} \tilde{\Theta}_{j, T-2}\right]
$$

with

$$
\begin{aligned}
\tilde{\Theta}_{j, T-2}= & \exp \left(a_{j} \alpha_{j, T-1}^{* \top} W_{T-1} R\right) \\
& \cdot \mathrm{E}_{T-2}\left[\left(1-p_{T-2}\right) \exp \left\{-a_{j}\left(\alpha_{j, T}^{* \top} \lambda_{T} W_{T}\right)\right\} \tilde{\Theta}_{j, T-1}+p_{T-2} \Theta_{j, T}\right] .
\end{aligned}
$$

By backward induction we obtain (17) and (18).

From (17) we observe that a non-zero issuer default probability influences adversely the demand for the WDs through correcting the expectations of the future portfolio values and the marginal expected utility of the WD payoffs downwards.

\subsection{Alternative Investment}

Frequently, the model assumptions implying that agents' portfolios contain only the risk free asset besides the WDs will not hold. This will often be the case for the investor's portfolio. In the following, we relax this restriction and allow investor to make an alternative investment on the financial market.

Let's amend the assumptions made in 2.1 with the following:

1a Assets. Let assumption 1. hold. Let $F_{t}$ be a quoted price of an exchange traded 
financial asset at time $t$. While $F_{t}$ is given, $F_{t+1}$ is random, bounded, and predictable at $t$. Trading with $F_{t}$ is not restricted in any way, that is, short and long positions in the asset in each $t$ are possible. We assume there is no transaction costs on the asset market. As before, no capital addition or withdrawals are possible throughout the investment horizon, such that the agents are exposed to self-financing constraints. For example, $F_{t}$ can be a share value of an exchange traded fund tracking some financial portfolio, or it can represent the value of the market portfolio itself at $t$.

2a Agents. Let assumption 2. hold. Now, issuer $m$ holds additionally $f_{m, t}$ shares of the exchange traded financial asset with exogenous price $F_{t}$. Also, $f_{\text {., }}$ is real valued, that is, all assets are perfectly divisible and short sales are allowed. The value of issuer's portfolio at time $t$ becomes $V_{m, t}=\alpha_{m, t}^{\top} W_{t}-f_{m, t} F_{t}+\beta_{m, t} B_{t}$.

As before, in each period $t$ of the investment horizon, agents maximise their expected utility of the terminal wealth with the available WDs and attain their demand and supply for the WDs. That is, in each period $t<T$ issuer $m$ determines her selffinancing trading strategy $\left(\alpha_{m, t+1}, \beta_{m, t+1}, f_{m, t+1}\right)_{t=0,1, \ldots, T}^{\top}$, in particular, she constructs the optimal hedging portfolio given the state of the system at time $t$. Partial market clearing with respect to WDs determines the equilibrium prices for the WDs.

3a State. Let assumption 3. hold. The observable state of the system for agent $i$ at time $t$, denoted by $\mathcal{W}_{i, t}$, contains additionally the quoted price $F_{t}$.

Under assumptions 1a-3a, the terminal wealth of investor $m$ at $T$ is:

$$
\Pi_{m, T}=-\alpha_{m, T}^{\top} W_{T}-f_{m, T} F_{T}+\beta_{m, T} B_{T}=V_{m, T}
$$

with $\alpha_{m, T}^{\top} W_{T}, f_{m, T} F_{T}$, and $\beta_{m, T} B_{T}$ being the payoffs of the WDs portfolio, the alternative 
financial investment, and the risk free asset respectively. Investor's portfolio choice problem in each $t=0,1, \ldots, T-1$ is:

$$
\begin{aligned}
& \max _{\alpha_{m, t+1: T} \in \mathbb{R}^{S \times(T-t)}} \mathrm{E}_{t}\left\{U_{m}\left(\Pi_{m, T}\right)\right\} \\
& \text { s.t. } \alpha_{m, t+1}^{\top} W_{t}+f_{m, t+1} F_{t}-\beta_{m, t+1} B_{t}+V_{m, t}=0 .
\end{aligned}
$$

As before, in each $t<T$ investor $m$ maximises the expected utility of her terminal wealth with respect to all future trading strategies, subject to a self-financing portfolio. The expected utility of the investor in $t J_{m, t}\left(V_{m, t}, \alpha_{m, t+1: T}, f_{m, t+1: T}, \mathcal{W}_{t}\right)$ is now also a function of her trading strategies on the financial market, denoted as $f_{m, t+1: T}$.

Keeping in mind these modifications and following the steps of subsection 2.2, we have to modify the investor's supply for WD $s$ in $(14)$ in the following way:

$$
\begin{aligned}
& W_{t, s}^{m}\left(\alpha_{m, t+1}, f_{m, t+1}\right)=\frac{\mathrm{E}_{t}\left[\exp \left\{a_{m} R^{T-(t+1)}\left(\alpha_{m, t+1}^{\top} W_{t+1, s}+f_{m, t+1} F_{t+1}\right) \Theta_{m, t} W_{t+1, s}\right\}\right]}{\mathrm{E}_{t}\left[\exp \left\{a_{m} R^{T-(t+1)}\left(\alpha_{m, t+1}^{\top} W_{t+1, s}+f_{m, t+1} F_{t+1}\right) \Theta_{m, t}\right\}\right] R}, \\
& J_{m, t}^{*}\left(V_{m, t}, \mathcal{W}_{m, t}\right)=-\exp \left(-a_{m} V_{m, t} R^{T-t}\right) \Theta_{m, t}, \\
& \Theta_{m, t}=\exp \left\{-a_{m} R^{T-t}\left(\alpha_{m, t+1}^{* \top} W_{t}+f_{m, t+1}^{*} F_{t}\right)\right\} \\
& \mathrm{E}_{t}\left[\exp \left\{a_{m} R^{T-t+1}\left(\alpha_{m, t+1}^{* \top} W_{t+1}+f_{m, t+1}^{*} F_{t}\right) \Theta_{m, t+1}\right\}\right]
\end{aligned}
$$

for $t=0, \ldots, T-1, \Theta_{m, T}=1$ and $R=1+r$. In $(33), f_{m, t+1}^{*}$ should be chosen such that it satisfies:

$$
F_{t}=\frac{\mathrm{E}_{t}\left[\exp \left\{a_{m} R^{T-(t+1)}\left(\alpha_{m, t+1}^{\top} W_{t+1, s}+f_{m, t+1}^{*} F_{t+1}\right) \Theta_{m, t} F_{t+1}\right\}\right]}{\mathrm{E}_{t}\left[\exp \left\{a_{m} R^{T-(t+1)}\left(\alpha_{m, t+1}^{\top} W_{t+1, s}+f_{m, t+1}^{*} F_{t+1}\right) \Theta_{m, t}\right\}\right] R},
$$

We obtained (34) by maximising $J_{m, t}\left(V_{m, t}, \alpha_{m, t+1: T}, f_{m, t+1: T}, \mathcal{W}_{m, t}\right)$ with respect to $f_{m, t+1: T}$ in each $t$ as in subsection 2.2 . 
Note, that now the reverse supply for the sth WD also depends on the position in the alternative financial investment. Thus, any changes in its price process will influence investor's supply for the sth WD.

\section{Pricing Weather Derivatives Using Weather Data}

In this section, we show an example on how to price rainfall derivatives using historical weather data from China and discuss other applications of the pricing approach presented.

\subsection{Pricing Chinese Rain}

Chinese farmers are exposed to pronounced weather risks as Turvey and Kong (2010). According to The World Bank (2007) the existing agricultural insurance schemes are too expensive for Chinese agricultural producers. One of the causes might be the fact that crop insurer are exposed to variability in precipitation due to the impact of the later on farmers' crop production. Trading WDs can play an important role in transferring part of the weather exposure to financial markets and so make crop insurance affordable for farmers. Since the weather exposed income of such a crop insurer is generally dependent on the rainfall in a number of geographical sites where the insured farmers are located, a basket of rainfall derivatives on the relevant sites should be used for hedging the risks. Motivated by this example, we illustrate pricing a basket of rainfall options on two weather stations Changde and Enshị located in an agricultural area of China.

\footnotetext{
${ }^{1}$ Station numbers given by the World Meteorological Organisation are 57662 for Changde and 57447 for Enshi.
} 


\subsubsection{Setup}

Our representative buyer is an insurer who offers crop insurance to the farmers in the agricultural area of Changde and Enshi is, therefore, exposed to fluctuations in the local rainfall amount. The company discovers that its income is highly dependent on the cumulative precipitation in Changde and Enshi during May, and wants to hedge its rainfall exposure by holding a portfolio of put options on the cumulative rainfall in these sites. Suppose, there is an investor who wants to issue such rainfall derivatives on the two of the relevant sites: Changde and Enshi. Then, the parties consider pricing a basket of the two put options on the cumulative precipitation over May in Changde and Enshi. They construct the put options as plain vanilla options on the underlying rainfall indices computed as the sums of monthly rainfall over May measured in each of the sites. Both options mature at the end of the measurement period, that is, on the 31st of May. We also suppose that the parties aggree to renegotiate the put prices in the middle of the measurement period: on the 15th of May.

Let $\mathcal{R}_{z, i}$ denote the rainfall measured at station $i$ on day $z$. The cumulative precipitation over a period $Z$ is then $\sum_{z \in Z} \mathcal{R}_{z, i}$. Thus, a put option on the cumulative precipitation in $Z$ in station $i$ with strike $K_{i}$ (in precipitation units) has the payoff:

$$
k \max \left\{K_{i}-\sum_{z \in Z} \mathcal{R}_{z, i}, 0\right\}
$$

where $k$ specifies the tick value of the option in monetary units.

Suppose, the insurer's income $I_{1}$ exhibits a non-linear dependence to the rainfall index in May of the form $I_{1} \sim \log \mathcal{N}\left\{\mu_{I}\left(R_{z, i}\right), \sigma_{I}\left(R_{z, i}\right)\right\}$ where $\mu_{I}\left(R_{z, i}\right)=10+\prod_{i \in 1,2} \sum_{z \in \text { May }} \mathcal{R}_{z, i} / K_{i}$ and $\sigma_{I}\left(R_{z, i}\right)=0.1\left\{1+\sum_{i \in 1,2}\left(1-\mathcal{R}_{z, i} / K_{i}\right)^{2}\right\}$ parametrize the dependence of $I_{1}$ to the rainfall 
of Changde (indexed by 1) and Enshi (indexed by 2) respectively. Further parameters to specify are: the risk aversion $a_{j}=a_{m}=10^{-4}$, the strikes $K_{1}=K_{2}=100$ and the tick value $k=1$ monetary units per mm precipitation measured.

Logarithmic return on the alternative investment in investor's portfolio is assumed to follow a normal distribution with zero mean and annual volatility $\sigma_{F}=0.1$ in the low volatility scenario and $\sigma_{F}=0.25$ in the high volatility scenario. The value of the alternative investment in $t=0$ is normalized to a hundred monetary units, that is $F_{0}=100$.

We compute the prices for the put options assuming different investment horizons $T$. If $T=1$, in $t=0$ (prior to the 1st of May) the agents negotiate the prices for the specified options under their current states of the world $\mathcal{W}_{1,0}$ and $\mathcal{W}_{2,0}$ respectively, and at $t=T$ (here, on the 31st of May) the settlement occurs according to the terminal time with realized payoffs $W_{T} . T=2$ means that the agents additionally renegotiate the prices at $t=1$ (here, on the 15th of May). The renegotiation at $t=1$ takes place under the new circumstances arising from their new states $\mathcal{W}_{1,1}$ and $\mathcal{W}_{2,1}$ respectively. The final payments are then settled according to the realizations of $W_{2}$. In our example, the state $\mathcal{W}_{i, t}, i=1,2$ includes the evolution of the rainfall, the obeserved price process $W_{n, n \leq t}, F_{t}, \lambda_{t}, p_{t}$, and $r$. The evolution to the next state $\mathcal{W}_{i, t+1}$ conditional on the realization of $\mathcal{W}_{i, t}$ is described by conditional distribution function $\Phi$. The terminal state of the insurer $\mathcal{W}_{1, T}$ contains also her realised income $I_{1}$.

At $t \leq T$ denote the index value underlying the payoff in (35) in station $i$ as $R X_{t, i}$. Then, $R X_{0, i}=0, R X_{T, i}=\sum_{z \in 1 .-31 . M a y} \mathcal{R}_{z, i}$, and in case $T=2 R X_{1, i}=\sum_{z \in 1 .-15 . M a y} \mathcal{R}_{z, i}$. The corresponding put option price, denoted as $W_{t, i}$, corresponds in $t=T$ to the put payoff, $W_{T, i}=\max \left(K_{i}-R X_{T, i}, 0\right)$, and for $t<T W_{t, i}$ it is defined by the intersection of buyer's demand (17) and investor's supply (14). 


\subsubsection{Generation of the correlated rainfall on a daily basis}

To conduct the pricing of the rainfall options in the setup described, we will need to assess the joint distribution of the rainfall in Change and Enshi on a daily basis. We use the multi-site rainfall generation model proposed by Wilks (1998). We give the basic ideas of the Wilks model below.

Precipitation at time $t$ in station $s^{\prime} \mathcal{R}_{s^{\prime}, t}$ is modeled as:

$$
\mathcal{R}_{s^{\prime}, t}=r_{s^{\prime}, t} X_{s^{\prime}, t},
$$

where $X_{s^{\prime}, t}$ takes values $\{0,1\}$ and represents rainfall occurrence at time $t$ in station $s^{\prime}$ and $r_{s^{\prime}, t}$ is a positive random variable for the rainfall amount. This structure ensures that $\mathcal{R}_{s^{\prime}, t}$ equals zero whenever $X_{s^{\prime}, t}$ is zero and there is no rain and $\mathcal{R}_{s^{\prime}, t}$ equals $r_{s^{\prime}, t}$ whenever rainfall occurs. $X_{s^{\prime}, t}$ are assumed to form a Markov chain with two states, wet and dry:

$$
X_{s^{\prime}, t}=\left\{\begin{array}{l}
1\left(\text { wet }, \geq X_{\min }\right), \\
0\left(\text { dry },<X_{\min }\right) .
\end{array}\right.
$$

We take a first order Markov model where the probability of a wet day depends only on the state in the previous day. Our justification of this choice is based on the Bayesian information criteria (BIC), see Katz (1981) and the Table 2, where the lowest value of the test statistics indicates the appropriate order of the markov chain. The transition probabilities to the wet state are:

$$
\begin{aligned}
& P\left(X_{s^{\prime}, t}=1 \mid X_{s^{\prime}, t-1}=0\right)=p_{01, s^{\prime}, t}, \\
& P\left(X_{s^{\prime}, t}=1 \mid X_{s^{\prime}, t-1}=1\right)=p_{11, s^{\prime}, t} .
\end{aligned}
$$


The multi-site feature is added to the model through the generation of correlated occurrences in the neighbor locations. We define the threshold probability $p_{s^{\prime}, \text { crit }}$ :

$$
p_{c r i t, s^{\prime}, t}=\left\{\begin{array}{l}
p_{01, s^{\prime}, t} \text { if } X_{s^{\prime}, t-1}=0, \\
p_{11, s^{\prime}, t} \text { if } X_{s^{\prime}, t-1}=1,
\end{array}\right.
$$

and $X_{s^{\prime}, t}$ can be generated using

$$
X_{s^{\prime}, t}=\left\{\begin{array}{l}
1 \text { if } w_{s^{\prime}, t} \leq \Phi^{-1}\left(p_{c r i t, s^{\prime}, t}\right), \\
0 \text { if } w_{s^{\prime}, t}>\Phi^{-1}\left(p_{c r i t, s^{\prime}, t}\right) .
\end{array}\right.
$$

Here $\Phi(\cdot)$ is cumulative distribution function of standard normal distribution, $\left\{w_{s, t}\right\}_{s \in \mathcal{S}} \sim$ $N\left(0_{\mathcal{S}}, \Sigma\right)$, with $\Sigma_{s, s^{\prime}}=\operatorname{Corr}\left(w_{s, t}, w_{s^{\prime}, t}\right)$ such that the empirical correlations $\operatorname{Corr}\left(X_{s, t}, X_{s^{\prime}, t}\right)$ of the rainfall occurrences are mimicked in the generated rainfall occurrence series, see Wilks (1998) for further details.

The multi-site rainfall amount conditioned on a rainy day $r_{s, t} \mid X_{s, t}=1$ follows a mixture of two exponential distributions with a time dependent mixing parameter $\gamma_{s, t}$ and time changing means $\beta_{1, s, t}, \beta_{2, s, t}$. Following Wilks (1998) we can generate rainfall amounts at time $t$ in site $s$ using

$$
r_{s, t}=r_{\text {min }}-\beta_{s, t} \log \Phi\left(v_{s, t}\right)
$$

where

$$
\beta_{s, t}=\left\{\begin{array}{l}
\beta_{1, s, t} \text { if } \Phi\left(w_{s, t}\right) / p_{s, c r i t} \leq \gamma_{s, t}, \\
\beta_{2, s, t} \text { if } \Phi\left(w_{s, t}\right) / p_{s, c r i t}>\gamma_{s, t}
\end{array}\right.
$$

and $v_{s, t}$ are normal covariates correlated such that the generated rainfall time series mimic the empirical correlation in the rainfall data. 
Now, we estimate the parameters for the multisite rainfall generation in Changde and Enshi based on the rainfall data collected in these locations. Table 1 summarizes the parameters of the daily rainfall data for Changde and Enshi acquired via Research Data Center of CRC 649 (Collaborative Research Center 649: Economic risk).

\begin{tabular}{cccccc}
\hline \hline station & number & latitude & longitude & start date & end date \\
\hline Changde & 57662 & 29.05 & 111.68 & 19510101 & 20091130 \\
Enshi & 57447 & 30.28 & 109.47 & 19510801 & 20091130
\end{tabular}

Table 1Description of the rainfall data and stations.

For the estimation we use maximum likelihood. For the acquired rainfall data $p_{01,1, t \in \text { May }}=$ 0.39 and $p_{11,1, t \in \text { May }}=0.59$ whereas $p_{01,2, t \in \text { May }}=0.43$ and $p_{11,2, t \in \text { May }}=0.64$ where the indices 1 and 2 refer to Changde and Enshi respectively. The empirical counter part of $\operatorname{Corr}\left(X_{1, t \in \text { May }}, X_{2, t \in \text { May }}\right)$ appears to be 0.53 , in order to mimique this correlation $\operatorname{Corr}\left(w_{1, t \in M a y}, w_{2, t \in M a y}\right)$ must to be set to 0.76 . The empirical counter part of $\operatorname{Corr}\left(r_{1, t \in \text { May }}, r_{2, t \in \text { May }} \mid X_{1, t \in \text { May }}=1, X_{2, t \in \text { May }}=1\right)$ is 0.16 for the considered data, and in order to obtain this correlation in the generated rainfall series $\operatorname{Corr}\left(v_{1, t \in \text { May }}, v_{2, t \in \text { May }}\right)$ must be 0.25 .

The estimated distributional parameters for $r_{i, t \in \text { May }} \mid X_{i, \in \text { May }}=1, i=\{1,2\}$ are presented in Table 3 ,

Table 2BIC criterion for different orders of Markov model for $X_{t, s}, t \in\left[\tau_{1}, \tau_{2}\right], s \in$ \{Changde, Enshi, Yichang\}.

\begin{tabular}{llcc}
\hline Order/BIC & Changde & Enshi & Yichang \\
\hline 0 & 70.83 & 60.02 & 19.86 \\
1 & 53.21 & 43.21 & 4.53 \\
2 & 53.47 & 44.69 & 9.03 \\
3 & 65.64 & 59.72 & 33.38 \\
\hline
\end{tabular}


Table 3The estimated parameters for the mixture of two exponential distributions.

\begin{tabular}{lcl}
\hline Parameter & Changde & Enshi \\
\hline$\gamma_{, t \in\left[\tau_{1}, \tau_{2}\right]}$ & 0.78 & 0.58 \\
$\beta_{1,, t \in\left[\tau_{1}, \tau_{2}\right]}$ & 15.90 & 23.14 \\
$\beta_{2,, t \in\left[\tau_{1}, \tau_{2}\right]}$ & 0.62 & 1.86 \\
\hline
\end{tabular}

Using the estimated parameters, we generate $10^{4}$ correlated rainfall paths in Changde and Enshi to compute the model prices in our Monte Carlo example.

\subsubsection{Results}

By altering $T, p, r$ and $\sigma_{F}$ we obtain prices on rainfall calls for Changde and Enshi under different market scenarios. All prices are normalized to the price obtained for $T=1, p=0$, $r=0.01$ and $\sigma_{F}=0.1$ to facilitate the comparison.

Table 4

Prices for put options on cumulative rainfall in different scenarios.

Prices are computed for plain vanilla put options on cumulative precipitation over May in Changde $\left(C R_{\text {May, } 1}\right)$ and Enshi $\left(C R_{M a y, 2}\right)$ with strike $K=100$ for variing investment horizon $(T)$, capital costs $(r)$, default probabilities $\left(p_{t}=p\right)$, and market volatility $\left(\sigma_{F}\right)$.

\begin{tabular}{lrrrrrr}
\hline \multicolumn{1}{c}{ Scenarios } & \multicolumn{2}{c}{ Put on $C R_{\text {May }, 1}$} & & \multicolumn{2}{c}{ Put on $C R_{\text {May }, 2}$} \\
& & $T=1$ & $T=2$ & & $T=1$ & $T=2$ \\
\hline$\sigma_{F}=0.1$ & & & & \\
\hline$r=1 \%$ & $p=0$ & 100.00 & 100.30 & & 100.00 & 96.68 \\
$r=5 \%$ & $p=0$ & 99.67 & 99.95 & & 98.81 & 96.35 \\
$r=1 \%$ & $p=0.05$ & 91.22 & 93.95 & & 86.74 & 87.45 \\
$r=5 \%$ & $p=0.05$ & 90.87 & 93.62 & & 85.95 & 87.12 \\
\hline$\sigma_{F}=0.25$ & & & & \\
\hline$r=1 \%$ & $p=0$ & 100.00 & 100.31 & & 100.23 & 96.68 \\
$r=5 \%$ & $p=0$ & 99.67 & 99.97 & & 99.71 & 96.36 \\
$r=1 \%$ & $p=0.05$ & 91.23 & 94.23 & 86.88 & 87.73 \\
$r=5 \%$ & $p=0.05$ & 90.92 & 93.99 & 86.47 & 87.51 \\
\hline
\end{tabular}




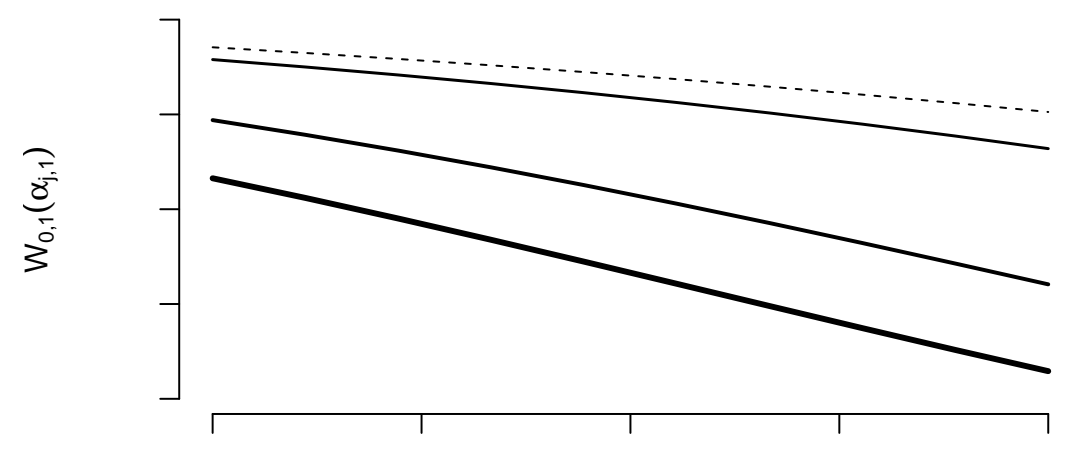

$\alpha_{j, 1,1}$

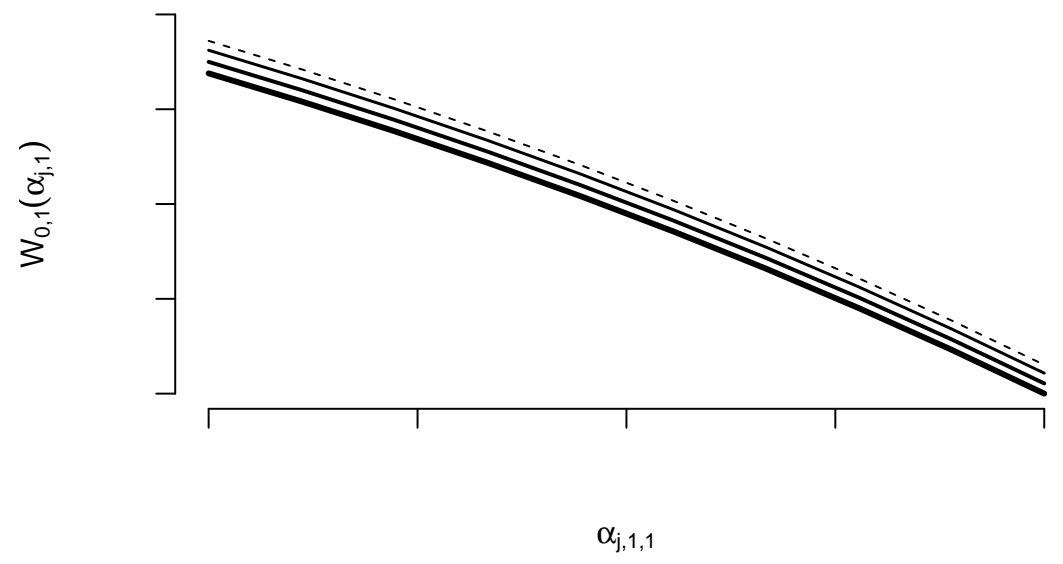

Figure 1. Shifts of the demand curves by increasing default risk (top) and capital costs (bottom). Top: the default risk probability $p_{t}=p$ is changing from 0 (dashed) to $0.01,0.05,0.10$ (solid lines, thicker with increasing $p$ ). Bottom: capital cost p.a. $r$ is changing from $1 \%$ (dashed) to $5 \%, 10 \%, 15 \%$ (solid lines, thicker with increasing $r$ ) for $\sigma_{F}=0.1$. Both plots: the $x$-axis shows $\alpha_{j, 1,1}$, the buyer's position in the put option on the rainfall in Changde during May $\left(C R_{M a y, 1}\right)$ with strike $K=100$ and investment horizon $T=1$; the $y$-axis shows $W_{0,1}\left(\alpha_{j, 1}\right)$, the price buyer is willing to pay for such an option, where $\alpha_{j, 1}=\left(\alpha_{j, 1,1}, \alpha_{j, 1,2}\right)^{\top}$ and while $\alpha_{j, 1,2}$, the position in the other put option on the rainfall in Enshi during May $\left(C R_{\text {May }, 2}\right)$ is kept constant. 
By a non-zero default probability the demand curve shifts downwards which results in lower equilibrium prices for the rainfall options. A higher risk-free rate $r$ also results in lower WD prices to compensate for higher capital costs.

The shift of the demand curve by changing probability to default and capital costs is shown in Figure 1.

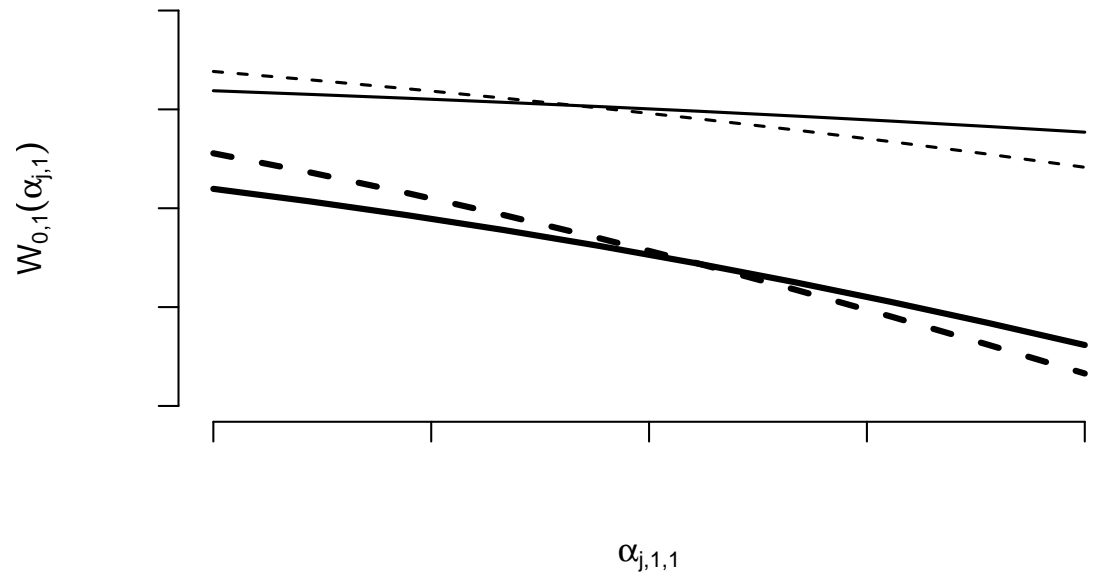

Figure 2. Shifts of the demand curves by increasing investment horizon $T$. Buyer's demand with investment horizon $T=1$ (dashed) and $T=2$ (solid). Thinner lines correspond to the case capital costs $r=5 \%$ and probability of issuer's default $p_{t}=p=0$; thicker lines correspond to capital costs $r=5 \%$ and probability of issuer's default $p_{t}=p=0.05$ for $\sigma_{F}=0.1$. The $x$-axis shows $\alpha_{j, 1,1}$, the buyer's position in the put option on the rainfall in Changde during May $\left(C R_{M a y, 1}\right)$ with strike $K=100$; the $y$-axis shows $W_{0,1}\left(\alpha_{j, 1}\right)$, the price buyer is willing to pay for such an option, where $\alpha_{j, 1}=\left(\alpha_{j, 1,1}, \alpha_{j, 1,2}\right)^{\top}$ and while $\alpha_{j, 1,2}$, the position in the other put option on the rainfall in Enshi during May $\left(C R_{M a y, 2}\right)$ is kept constant.

Figure 2 shows buyer's demand in $t=0$ for different time horizons $T$. In the "flexible" case $T=2$ the WD prices are renegotiated at $t=1$, and in the case $T=1$ no rebalancing takes place. From Figure 2 we observe that buyer's demand price elasticity at $t=0$ is 
lower in the "flexible" case. Consistent with the classical result of Allen and Postlewaite (1984), already at $t=0$ individual demand reflects all agents' expectations including the expectations about future trading behaviour.

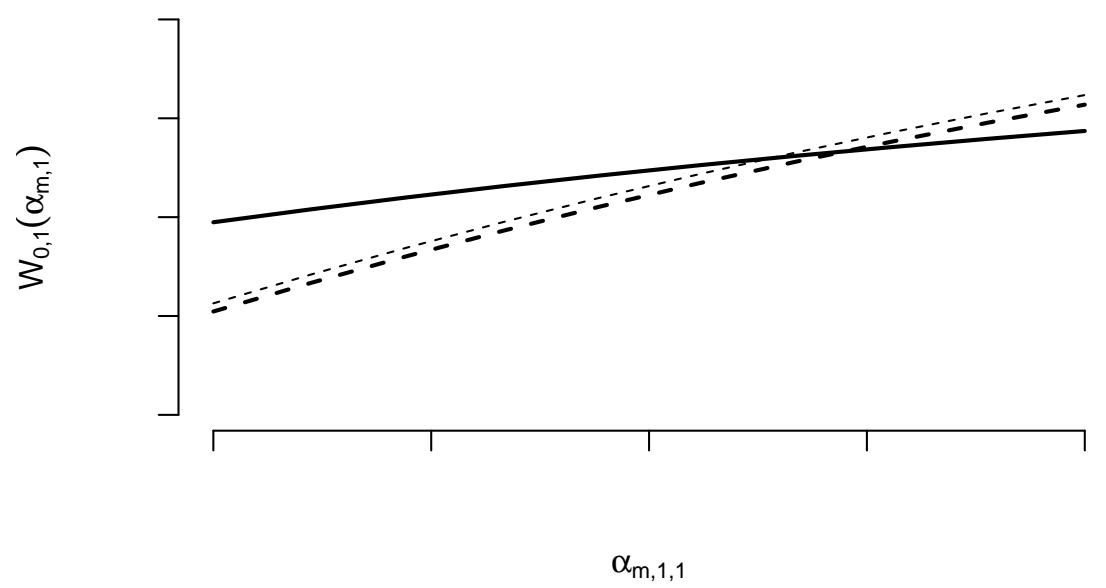

Figure 3. Shifts of the supply curves by increasing volatility of the alternative investment. Investor's supply with investment horizon $T=1$ (dashed) and $T=2$ (solid). Thinner lines correspond to the case of low volatility of the alternative investment; thicker lines correspond to high volatility. The $x$-axis shows $\alpha_{m, 1,1}$, the investor's position in the put option on the rainfall in Changde during May $\left(C R_{M a y, 1}\right)$ with strike $K=100$; the $y$-axis shows $W_{0,1}\left(\alpha_{m, 1}\right)$, the price for which the investor is willing to sell such an option, where $\alpha_{m, 1}=\left(\alpha_{m, 1,1}, \alpha_{m, 1,2}\right)^{\top}$ and while $\alpha_{m, 1,2}$, the position in the other put option on the rainfall in Enshi during April and May $\left(C R_{M a y, 2}\right)$ is kept constant.

Figure 3 shows investor's supply in $t=0$ for different time horizons $T$ and for different levels in the volatility of the alternative investment in investor's portfolio. In the "flexible" case $T=2$ the WD prices are renegotiated at $t=1$, and in the case $T=1$ no rebalancing takes place. From Figure 3 we observe that the price elasticity of the investor's supply at $t=0$ is lower in the "flexible" case. Moreover, the reaction of the supply to the changes 
in market volatility is very subtle in the "flexible" case (under $1 \%$ and is not visible in the graph), as the downward shift of the supply curve is quite pronounced for $T=1$. In this case, increase in market volatility (or in the volatility of the alternative investment) significantly stimulates the investor's supply for WDs.

\subsection{Discussion}

Our example shows pricing rainfall options. The presented pricing model, however, is not limited to any particular kind of weather derivatives. In fact, it is possible to price various weather derivatives with such underlyings as snowfall, sunshine hours, number of sunny days, number of rainy days, wind speed, and other weather indices of a practical relevance for retail, tourism and renewable energy operators.

Along with the other assumptions, the premise is the existence of a probabilistic model that precisely enough describes the distribution and the time evolution of the underlying weather index. Then, the conditional expectations of agents' utilities, which determine their supply and demand, can be approximated using Monte Carlo samples from the fitted probabilistic model. Applying partial market clearing condition gives the equilibrium prices for the constructed WDs.

\section{Summary}

We derive a dynamic utility-based model for pricing baskets of weather derivatives on multiple dependent underlyings. Via expected utility maximisation, dynamic portfolio optimisation over a finite investment horizon, and partial market clearing, we obtain semi-closed forms for the equilibrium prices of weather derivatives and for the optimal trading strategies. 
We provide extensions of the model to account for counterparty default risk and a possibility of an alternative financial investment.

As expected, there is an adverse effect of the increasing default risk and capital costs on the demand for weather derivatives and on their prices. We find, however, a stimulating effect of increasing market volatility on the supply for weather derivatives.

We apply the proposed model to price the rainfall options using the rainfall data of agricultural provinces Changde and Enshi in China. We compare the equilibrium prices for put options on cumulative rainfall over May resulting from different scenarios. The effects of increasing default risk, capital costs, investment horizon, and market volatility are assessed.

Applicability of the proposed model is not restricted to pricing rainfall derivatives. Various kind of weather derivatives with agent-based market design can be priced in our framework. 


\section{References}

Alaton, P., Djehiche, B., and Stillberger, D. (2002). On modelling and pricing weather derivatives. Applied Mathematical Finance, 1(9):1-20.

Allen, F. and Postlewaite, A. (1984). Rational expectations and the measurement of a stock's elasticity of demand. Journal of Finance, 39(4):1119-1125.

Benth, F. E., S̆altyté Benth, J., and Koekebakker, S. (2007). Putting a price on temperature. Scandinavian Journal of Statistics, 34(4):746-767.

Cao, M. and Wei, J. (2004). Pricing weather derivative: an equilibrium approach. Journal of Futures Markets, 24:1065-1089.

Carmona, R. and Diko, P. (2005). Pricing precipitation based derivatives. International Journal of Theoretical and Applied Finance, 8(7):959-988.

Çanakoğlu, E. and Özekici, S. (2009). Portfolio selection in stochastic markets with exponential utility functions. Annals of Operations Research, 166(1):281-297.

Chaumont, S., Imkeller, P., and Mï $\frac{1}{2}$ ller, M. (2006). Equilibrium trading of climate and weather risk and numerical simulation in a markovian framework. Stochastic Environment Research and Risk Assessement, 20:184-205.

Glauber, J., Collins, K., and Barry, P. (2002). Crop insurance, disaster assistance, and the role of the federal government in providing catastrophic risk protection. Agricultural Finance Review, 62:81-101. 
Golden, L., Wang, M., and Yang, C. (2007). Handling weather related risks through the financial markets: considerations of credit risk, basis risk, and hedging. Journal of Risk E Insurance, 74(2):319-346.

Heath, D., Jarrow, R., and Morton, A. (1992). Bond pricing and the term structure of inerest rates: a new methodology for contigent claims valuation. Econometrica, 60(1):77-105.

Horst, U. and Müller, M. (2007). On the spanning property of risk bonds priced by equilibrium. Mathematics of Operation Research, 32(4):784-807.

Hull, J. and White, A. (1995). The impact of default risk on the prices of options and other derivative securities. Journal of Banking \& Finance, 19(2):299-322.

Jarrow, R. A. and Turnbull, S. M. (1995). Pricing derivatives on financial securities subject to credit risk. Journal of Finance, 50(1):53-85.

Katz, R. (1981). On some criteria for estimating the order of a markov chain. Technometrics, 23(3):243-249.

Lee, Y. and Oren, S. (2010). A multi-period equilibrium pricing model of weather derivatives. Energy Systems, 1:3-30.

Leobacher, G. and Ngare, P. (2011). On modelling and pricing rainfall derivatives with seasonality. Applied Mathematical Finance, 18(1):71-91.

López Cabrera, B., Odening, M., and Ritter, M. (2013). Pricing rainfall futures at the CME. Journal of Banking \&3 Finance, 37(11):4286-4298.

Lucas, R. E. (1978). Asset prices in an exchange economy. Econometrica, 46(6):1429-1445. 
Musshoff, O., Odening, M., and Xu, W. (2010). Management of climate risks in agriculture - will weather derivatives permeate? Applied Economics, 43:1067-1077.

Pennacchi, G. (2008). Theory of Asset Pricing. Pearson Addison-Wesley, Boston.

Peréz-González, F. and Yun, H. (2013). Risk management and firm value: Evidence from weather derivatives. Journal of Finance, 68(5).

Stokey, N., Lucas, R., and Prescott, E. (1989). Recursive Methods in Economic Dynamics. Harvard University Press, Cambridge, MA.

The World Bank (2007). China: Innovations in agricultural insurance - promoting access to agricultural insurance for small farmers. Technical report, The World Bank.

Turvey, C. and Kong, R. (2010). Weather risk and the viability of weather insurance in china's gansu, shaanxi and henan provinces. China Agricultural Economic Review, 2:5-24.

Wilks, D. (1998). Multisite generalization of a daily stochastic precipitation generation model. Journal of Hydrology, 210(1-4):178 - 191.

Wu, Y.-C. and Chung, S.-L. (2010). Catastrophe risk management with counterparty risk using alternative instruments. Insurance: Mathematics and Economics, 47(2):234-245. 


\section{Appendix}

Proof of Proposition 1: We prove the assertion by backward induction starting with $t=T-1$. Utility maximisation problem of buyer $j$ in $T-1$ :

$$
\begin{aligned}
& \max _{\alpha_{j, T} \in \mathbb{R}^{S}} J_{j, T-1}\left(V_{j, T-1}, \alpha_{j, T}, \mathcal{W}_{j, T-1}\right) \\
& \text { s.t. } \alpha_{j, T}^{\top} W_{T-1}+\beta_{j, T} B_{T-1}-V_{j, T-1}=0 .
\end{aligned}
$$

The expected utility in $T-1$ reads:

$$
J_{j, T-1}\left(V_{j, T-1}, \alpha_{j, T}, \mathcal{W}_{j, T-1}\right)=\mathrm{E}_{T-1}\left\{-\exp \left(-a_{j} \Pi_{j, T}\right)\right\}
$$

By plugging the self-financing constraint in 44) we obtain

$$
J_{j, T-1}\left(V_{j, T-1}, \alpha_{j, T}, \mathcal{W}_{j, T-1}\right)=\mathrm{E}_{T-1}\left[-\exp \left\{-a_{j}\left(I_{j}+\alpha_{j, T}^{\top} W_{T}+R V_{j, T-1}-R \alpha_{j, T}^{\top} W_{T-1}\right)\right\}\right] .
$$

Taking the first derivative of (45) with respect to $\alpha_{j, T}$, we win the gradient vector with the sth entry equal to:

$$
\begin{aligned}
\frac{\partial J_{j, T-1}\left(V_{j, T-1}, \alpha_{j, T}, \mathcal{W}_{j, T-1}\right)}{\partial \alpha_{j, T, s}}= & a_{j} \exp \left\{-a_{j}\left(V_{j, T-1}-\alpha_{j, T}^{\top} W_{T-1}\right) R\right\} \\
& \cdot \mathrm{E}_{T-1}\left[\exp \left\{-a_{j}\left(I_{j}+\alpha_{j, T}^{\top} W_{T}\right)\right\}\left(W_{T, s}-R W_{T-1, s}\right)\right]
\end{aligned}
$$

for $s=1, \ldots, S$. Taking the second derivative of (45) with respect to $\alpha_{j, T}$ gives the Hessian 
matrix $H$ with $s s^{\prime}$ th entry:

$$
\begin{aligned}
& \frac{\partial^{2} J_{j, T-1}\left(V_{j, T-1}, \alpha_{j, T}, \mathcal{W}_{j, T-1}\right)}{\partial \alpha_{j, T, s} \partial \alpha_{j, T, s^{\prime}}}=-a_{j}^{2} \exp \left\{-a_{j}\left(V_{j, T-1}-\alpha_{j, T}^{\top} W_{T-1}\right) R\right\} \\
& \cdot \mathrm{E}_{T-1}\left[\exp \left\{-a_{j}\left(I_{j}+\alpha_{j, T}^{\top} W_{T}\right)\right\}\left(W_{T, s}-R W_{T-1, s}\right)\left(W_{T, s^{\prime}}-R W_{T-1, s^{\prime}}\right)\right]
\end{aligned}
$$

for $s, s^{\prime}=1, \ldots, S$. In a similar manner as Çanakoğlu and Özekici (2009), we check that the Hessian $H$ defined entry-wise by 47 is negative semi-definite, since for any nonzero $x \in \mathbb{R}^{S}$ :

$$
\begin{aligned}
x^{\top} H x= & -a_{j}^{2} \exp \left\{-a_{j}\left(V_{j, T-1}-\alpha_{j, T}^{\top} W_{T-1}\right) R\right\} \\
& \cdot \mathrm{E}_{T-1}\left[\exp \left\{-a_{j}\left(I_{j}+\alpha_{j, T}^{\top} W_{T}\right)\right\}\left\{\sum_{s=1}^{S} x_{s}\left(W_{T, s}-R W_{T-1, s}\right)\right\}^{2}\right]
\end{aligned}
$$

is always smaller or equal to zero. Hence, we obtain the maximiser of (45) by setting the gradient (46) coordinate-wise to zero, in particular:

$$
\mathrm{E}_{T-1}\left[\exp \left\{-a_{j}\left(I_{j}+\alpha_{j, T} W_{T}\right)\right\}\left(W_{T, s}-R W_{T-1, s}\right)\right]=0 .
$$

In $T-1$ the current prices $W_{T-1}$ are known and can be taken out of the expectation in (49). Thus, we obtain the reverse demand of the $j$ th buyer for each $s \in \mathcal{S}$ as:

$$
W_{T-1, s}^{j}\left(\alpha_{j, T}\right)=\frac{\mathrm{E}_{T-1}\left[\exp \left\{-a_{j}\left(I_{j}+\alpha_{j, T}^{\top} W_{T}\right)\right\} W_{T, s}\right]}{\mathrm{E}_{T-1}\left[\exp \left\{-a_{j}\left(I_{j}+\alpha_{j, T}^{\top} W_{T}\right)\right\}\right] R}=\frac{\mathrm{E}_{T-1}\left\{\exp \left(-a_{j} \alpha_{j, T}^{\top} W_{T}\right) \Theta_{j, T} W_{T, s}\right\}}{\mathrm{E}_{T-1}\left\{\exp \left(-a_{j} \alpha_{j, T}^{\top} W_{T}\right) \Theta_{j, T}\right\} R}
$$

with $\Theta_{j, T}=\exp \left(-a_{j} I_{j}\right)$.

Applying partial market clearing or zero-net-supply condition to all WDs, we find $\alpha_{j, T}^{*}$ 
and $W_{T-1}=W_{T-1}^{m}\left(\alpha_{m, T}^{*}\right)$. Then, the maximised utility of buyer $j$ at $T-1$ is:

$$
\begin{aligned}
J_{j, T-1}^{*}\left(V_{j, T-1}, \mathcal{W}_{j, T-1}\right) & =J\left\{V_{j, T-1}, \alpha_{j, T}^{*}, W_{T-1}, \mathcal{W}_{j, T-1}\right\} \\
& =-\exp \left\{-a_{j} V_{j, T-1} R\right\} \Theta_{j, T-1}
\end{aligned}
$$

with

$$
\Theta_{j, T-1}=\exp \left\{a_{j} \alpha_{j, T}^{* \top} W_{T-1} R\right\} \mathrm{E}_{T-1}\left[\exp \left\{-a_{j}\left(\alpha_{j, T}^{* \top} W_{T}\right)\right\} \Theta_{j, T}\right]
$$

$J_{j, T-1}^{*}\left(V_{j, T-1}, \mathcal{W}_{j, T-1}\right)$ is of the exponential form like the utility function itself, and the induction hypothesis holds for $t=T-1$. Assume, that it holds for $t=T, T-1, \ldots, k$. Then, in $k-1$ :

$$
\begin{aligned}
& \max _{\alpha_{j, k} \in \mathbb{R}^{S}} J_{j, k-1}\left\{V_{j, k-1}, \alpha_{j, k}, \mathcal{W}_{j, k-1}\right\} \\
& \text { s.t. } \alpha_{j, k}^{\top} W_{k-1, s}+\beta_{j, k} B_{k-1}-V_{j, k}=0 .
\end{aligned}
$$

is the constrained utility maximisation problem faced by buyer $j$. The expected utility in $k-1$ is:

$$
\begin{aligned}
J_{j, k-1}\left\{V_{j, k-1}, \alpha_{j, k}, \mathcal{W}_{j, k-1}\right\} & =\mathrm{E}_{k-1}\left\{J_{j, k}^{*}\left(V_{j, k}, \mathcal{W}_{j, k}\right)\right\} \\
& =\mathrm{E}_{k-1}\left\{-\exp \left(-a_{j} V_{j, k} R^{T-k}\right) \Theta_{j, k}\right\},
\end{aligned}
$$

where

$$
\Theta_{j, k}=\exp \left(a_{j} R^{T-k} \alpha_{j, k+1}^{* \top} W_{k}\right) \mathrm{E}_{k}\left\{\exp \left(-a_{j} R^{T-(k+1)} \alpha_{j, k+1}^{* \top} W_{k+1, s}\right) \Theta_{j, k+1}\right\} .
$$


Now we use the following identity:

$$
V_{j, k}=R\left(V_{j, k-1}-\alpha_{j, k}^{\top} W_{k-1}\right)+\alpha_{j, k}^{\top} W_{k}
$$

to rewrite (54) as a function of $\alpha_{j, k}$ :

$$
J_{j, k-1}\left(V_{j, k-1}, \alpha_{j, k}, \mathcal{W}_{j, k-1}\right)=\mathrm{E}_{k-1}\left(-\exp \left[-a_{j}\left\{R\left(V_{j, k-1}-\alpha_{j, k}^{\top} W_{k-1}\right)+\alpha_{j, k}^{\top} W_{k}\right\} R^{T-k}\right] \Theta_{j, k}\right)
$$

By taking the derivative of (57) with respect to $\alpha_{j, k}$ we find the gradient with sth entry:

$$
\begin{aligned}
& \frac{\partial J_{j, k-1}\left(V_{j, k-1}, \alpha_{j, k}, \mathcal{W}_{j, k-1}\right)}{\partial \alpha_{j, k, s}}=a_{j} R^{T-k} \exp \left\{-a_{j}\left(V_{j, k-1}-\alpha_{j, k}^{\top} W_{k-1}\right) R^{T-k+1}\right\} \\
& \cdot \mathrm{E}_{k-1}\left\{-\exp \left(-a_{j} \alpha_{j, k}^{\top} W_{k} R^{T-k}\right) \Theta_{j, k}\left(W_{k, s}-R W_{k-1, s}\right)\right\}
\end{aligned}
$$

As in (48) the Hessian is also negative semi-definite. Thus, the maximiser of (57) is found by setting the gradient $(58)$ to zero, that is:

$$
\mathrm{E}_{k-1}\left\{-\exp \left(-a_{j} \alpha_{j, k}^{\top} W_{k} R^{T-k}\right) \Theta_{j, k}\left(W_{k, s}-R W_{k-1, s}\right)\right\}=0
$$

We obtain the reverse demand of the $j$ th buyer for each $s \in \mathcal{S}$ as:

$$
W_{k-1, s}^{j}\left(\alpha_{j, k}\right)=\frac{\mathrm{E}_{k-1}\left\{\exp \left(-a_{j} \alpha_{j, k}^{\top} W_{k} R^{T-k}\right) \Theta_{j, k} W_{k, s}\right\}}{\mathrm{E}_{k-1}\left\{\exp \left(-a_{j} \alpha_{j, k}^{\top} W_{k} R^{T-k}\right) \Theta_{j, k}\right\} R}
$$

Partial market clearing in $k-1$ determines $\alpha_{j, k}^{*}, W_{k-1}=W_{k-1}^{m}\left(\alpha_{m, k}^{*}\right)$ and the maximised 
utility of buyer $j$ in this period:

$$
\begin{aligned}
J_{j, k-1}^{*}\left(V_{j, k-1}, \mathcal{W}_{j, k-1}\right)= & J_{j, k-1}\left\{V_{j, k-1}, \alpha_{j, k}^{*}, \mathcal{W}_{j, k-1}\right\} \\
& =-\exp \left\{-a_{j} V_{j, k-1} R\right\} \Theta_{j, k-1}
\end{aligned}
$$

with

$$
\Theta_{j, k-1}=\exp \left\{a_{j} \alpha_{j, k}^{* \top} W_{k-1} R\right\} \mathrm{E}_{k-1}\left[\exp \left\{-a_{j}\left(\alpha_{j, k}^{* \top} W_{k}\right)\right\} \Theta_{j, k}\right]
$$

which completes the proof. 


\section{SFB 649 Discussion Paper Series 2017}

For a complete list of Discussion Papers published by the SFB 649, please visit http://sfb649. wiwi.hu-berlin.de.

001 "Fake Alpha" by Marcel Müller, Tobias Rosenberger and Marliese UhrigHomburg, January 2017.

002 "Estimating location values of agricultural land" by Georg Helbing, Zhiwei Shen, Martin Odening and Matthias Ritter, January 2017.

003 "FRM: a Financial Risk Meter based on penalizing tail events occurrence" by Lining $\mathrm{Yu}$, Wolfgang Karl Härdle, Lukas Borke and Thijs Benschop, January 2017.

004 "Tail event driven networks of SIFIs" by Cathy Yi-Hsuan Chen, Wolfgang Karl Härdle and Yarema Okhrin, January 2017.

005 "Dynamic Valuation of Weather Derivatives under Default Risk" by Wolfgang Karl Härdle and Maria Osipenko, February 2017.

SFB 649, Spandauer Straße 1, D-10178 Berlin http:/ / sfb649.wiwi.hu-berlin.de 